\title{
Immunophenotypic characterization and depletion of pulmonary intravascular macrophages of horses
}

\author{
Om P. PARBHAKAR ${ }^{\mathrm{a}}$, Tanya DuKE ${ }^{\mathrm{b}}$, Hugh G.G. TOWNSEND ${ }^{\mathrm{c}}$, \\ Baljit SINGH ${ }^{\mathrm{a} *}$ \\ a Departments of Veterinary Biomedical Sciences, Western College of Veterinary Medicine, \\ 52 Campus Drive, University of Saskatchewan, Saskatoon, SK S7N 5B4, Canada \\ b Small Animal Clinical Sciences, Western College of Veterinary Medicine, 52 Campus Drive, \\ University of Saskatchewan, Saskatoon, SK S7N 5B4, Canada \\ ${ }^{c}$ Large Animal Clinical Sciences, Western College of Veterinary Medicine, 52 Campus Drive, \\ University of Saskatchewan, Saskatoon, SK S7N 5B4, Canada
}

(Received 10 February 2003; accepted 30 June 2003)

\begin{abstract}
Pulmonary intravascular macrophages (PIMs) are present in horses and are believed to increase their sensitivity to endotoxin-induced cardio-pulmonary shock. However, owing to a lack of a marker for PIMs and the inability to isolate them, their precise contributions in the horse remain unknown. We designed this study to identify an immuno-phenotypic marker for PIMs and to develop a protocol for their transient depletion with gadolinium chloride (GC). GC is a lanthanide that has been used to deplete liver and lung macrophages. The horses $(N=15)$ were divided into control $(n=5)$ and GC-treated $(n=10)$ groups and the lung samples were examined by routine and immunocytochemical light and electron microscopy. GC-treated horses were euthanized at $48 \mathrm{~h}$ $(n=6)$ and $72 \mathrm{~h}(n=4)$ post-treatment. The PIMs reacted with MAC-387 but not with ED-1 and CD-68 anti-macrophage antibodies. GC reduced the number of PIMs in horses at 48 and $72 \mathrm{~h}$ compared with the control $(p<0.05)$. There were increased intravascular TUNEL-positive cells in GC-treated horses and electron microscopy showed apoptotic PIMs in these horses. These data show that MAC-387 is a reliable marker for PIMs and GC is a safe tool to reduce the number of PIMs.
\end{abstract}

PIMs / gadolinium / MAC-387 / apoptosis / immunohistology / immuno-electron microscopy / TUNEL

\section{INTRODUCTION}

Macrophages are widely distributed in the body and display structural and functional heterogeneity [13]. In the lungs, macrophages occur in the airways, alveolar spaces, interstitium, and microvessels. Pulmonary intravascular macrophages (PIMs) were recently identified as resident mononuclear phagocytes in cattle, sheep, goats, pigs and horses [4, 5, 7, 29, 35]. PIMs differentiate from monocytes in post-natal life and form firm adhesions with the capillary endothelium [15, 36-38]. These cells phagocytize blood-born tracer particles, bacteria and endotoxins [3, 18, 27, 29, 36]. PIMcontaining species show pulmonary hypertension following the administration of tracer particles and endotoxins, and the removal of PIMs suppresses these responses

* Corresponding author: baljit.singh@usask.ca 
$[18,31,33]$. Neonatal lambs that do not have PIMs show diminished pulmonary vascular response to tracers compared with four-week old lambs that have PIMs [15]. Blecha et al. showed that porcine PIMs secrete thromboxane and are cytolytic [7, 8]. They also reported that the immunological characteristics of PIMs are similar to those of alveolar macrophages [8]. There is a putative association between the presence of PIMs and the susceptibility to acute lung disease $[32,35]$.

Equine PIMs show structural and functional similarities to their counterparts in other species [5, 29]. Halothane exposure activates equine PIMs as indicated by hyperplastic Golgi complexes and the development of lamellipods [2]. Horses show increases in respiration rate, heart rate and pulmonary arterial pressures following infusions of tracer particles or endotoxins [29]. Endotoxin-provoked increases in mean pulmonary arterial pressure are abolished by the pre-treatment of horses with a detergent called Tyloxapol $[16,18]$. Although it is believed that the detergent blocks the PIMs, the mechanisms of these effects remain unknown [17].

Acute lung inflammation is a critical factor in colic-associated equine deaths. These deaths cause serious suffering and substantial financial loss to the equine industry [20]. There is a suggestion that PIMs may play a critical role in acute lung inflammation in horses [17]. However, the functions of PIMs remain unknown largely for technical reasons. First, most attempts to isolate PIMs for in vitro investigations have failed because of their intimate attachment to the lung microvascular endothelium [26]. Second, there is a lack of a phenotypic marker to identify PIMs in situ. Third, it is difficult to attribute particular functions to an individual immune cell in vivo because many cells may contribute to an observed response. Now, we report data on the in situ phenotypic characterization of equine PIMs and a method to reduce their numbers in vivo.

\section{MATERIALS AND METHODS}

\subsection{Animals}

The experimental protocols for this study were approved by the Animal Care Committee of the University of Saskatchewan and the work was carried out according to the guidelines of the Canadian Council on Animal Care. Horses $(N=15)$ of 2-4 years of age, purchased from the local market, were acclimatized to the changed housing for over a week and were regularly physically examined. The horses were randomly divided into two groups: The control $(n=5)$ and the gadolinium chloride (GC, Sigma Co., USA) treated group $(n=10)$. GC is a heavy metal lanthanide that has previously been used to remove liver and lung macrophages including the PIMs [25, 28, 39]. In vitro studies show that GC induces apoptosis in macrophages [19].

\subsection{Pressure measurements}

The day before study, horses were sedated with xylazine hydrochloride (Rompun, Bayer Inc., Canada, $0.5 \mathrm{mg} / \mathrm{kg}$ body weight, IV) and under local anesthesia an $8 \mathrm{Fr}$ catheter introducer (Sheath Dilator, Arrow International Inc., USA) was aseptically positioned and secured within the right jugular vein. Next a 7 Fr, $110 \mathrm{~cm}$ thermistor tipped, triple lumen pulmonary artery balloon catheter (Edwards Swan-Ganz Catheter, Baxter Corp., Canada) was positioned so that its tip was in the pulmonary artery. The catheter location was confirmed by observation of characteristic blood pressure waveforms. The systolic, mean and diastolic pulmonary arterial blood pressures were measured using a silicon chip strain gauge transducer (Truwave Disposable Pressure Transducer, Baxter Corp., USA), and displayed on a physiological monitor (Propaq 104, Protocol Systems Inc., Canada). The transducer was zeroed to a point approximately level with the 
right atrium of the horse and was previously calibrated using a mercury manometer. Blood samples were collected for differential white cell counts.

\subsection{Treatments}

Control horses $(n=5)$ were administered $500 \mathrm{~mL}$ of physiological saline intravenously for $30 \mathrm{~min}$. Treated horses were given GC (Sigma Co., USA) intravenously at the dose of $10 \mathrm{mg} / \mathrm{kg}$ body weight in $500 \mathrm{~mL}$ of physiological saline solution over a $30 \mathrm{~min}$ period. Pulmonary arterial pressures were recorded three times: immediately before the infusions, after $50 \%$ and $100 \%$ of the infusions. Horses treated with GC were euthanized with pentobarbital sodium (Euthenol ${ }^{\circledR}$, Bimeda-MTS Animal Health Inc., Canada, $10 \mathrm{~mL} / 50 \mathrm{~kg}$ body weight intravenously) at $48 \mathrm{~h}(n=6)$ and $72 \mathrm{~h}(n=4)$ after treatment while the control horses were euthanized at $48 \mathrm{~h}$.

\subsection{Tissue fixation}

Following euthanasia, the horses were hoisted off the floor with chains in the necropsy room and the tracheae were exteriorized and opened. Five liters of fixative (2\% paraformaldehyde and $0.1 \%$ glutaraldehyde in $0.1 \mathrm{M}$ sodium cacodylate buffer; $\mathrm{pH}$ 7.2) were poured into the lungs via the tracheae and left in place for $30 \mathrm{~min}$. Then, the lungs were removed en bloc and divided into pre-determined imaginary slices to collect seven pieces $\left(2 \mathrm{~cm}^{3}\right)$ from each lung.

\subsection{Tissue processing}

\subsubsection{Light microscopy}

The tissues were fixed in $4 \%$ paraformaldehyde for $24 \mathrm{~h}$. These tissues were dehydrated in ascending concentrations of ethanol and xylene and embedded in paraffin.

\subsubsection{Routine electron microscopy}

Lung tissues were processed for EM as described previously [5]. Briefly, the tis- sues were fixed in $2 \%$ paraformaldehyde and $2.5 \%$ glutaraldehyde in $0.1 \mathrm{M}$ sodium cacodylate buffer ( $\mathrm{pH} 7.2$ ) for $3 \mathrm{~h}$ and postfixed for $90 \mathrm{~min}$ in $1.5 \%$ osmium tetroxide. The tissues were stained en bloc with $0.5 \%$ tannic acid in $0.1 \mathrm{M} \mathrm{HCl}$-sodium cacodylate for $30 \mathrm{~min}$, dehydrated and embedded in Jembed 812 resin (J.B. EM Services Inc., USA).

\subsubsection{Immuno-electron microscopy}

The lung pieces were fixed in $2 \%$ paraformaldehyde and $0.1 \%$ glutaraldehyde in $0.1 \mathrm{M}$ sodium cacodylate buffer $(\mathrm{pH} 7.2)$ for $3 \mathrm{~h}$ at $4{ }^{\circ} \mathrm{C}$ but were not treated with osmic acid. After dehydration, the tissues were infiltrated with LRWhite (J.B. EM Services Inc., USA) and polymerized in ultraviolet light at $-8{ }^{\circ} \mathrm{C}$ for $48 \mathrm{~h}$.

\subsection{Tissue analyses}

\subsubsection{Histopathology}

5-7 $\mu \mathrm{m}$ sections were cut from all the blocks (7/lung) and stained with hematoxylin and eosin.

\subsubsection{Immunohistology with ED-1, MAC-387 and CD68 antibodies}

5-7 $\mu \mathrm{m}$ thick sections were prepared from the paraffin-embedded lung tissues and placed on silane-coated glass slides. After removal of wax with xylene and rehydration in an ethanol series, the sections were treated with pepsin $(2 \mathrm{mg} / \mathrm{mL} 0.01 \mathrm{~N}$ $\mathrm{HCl}$ ) for $90 \mathrm{~min}$ for antigen retrieval. The sections were incubated in 5\% hydrogen peroxide for $20 \mathrm{~min}$ to quench endogenous peroxidase and with $1 \%$ bovine serum albumin for $30 \mathrm{~min}$ to block non-specific binding. We tested three anti-macrophage antibodies. ED-1 (Serotec Ltd., UK) is raised in the mouse and reacts with a $90-100 \mathrm{kDa}$ protein expressed on lysosomal membranes of rat macrophages and has been used to 
recognize them in flow cytometry and in routinely fixed tissues [24]. Mouse antihuman monocyte/macrophage antibody (MAC-387, Serotec Ltd., UK) recognizes the $\mathrm{L} 1$ cytoplasmic molecule and has been used in immunohistochemistry [11, 12]. CD68 (DAKO A/S, Denmark) is a mouse anti-human macrophage antibody that reacts with a $110 \mathrm{kDa}$ glycoprotein in tissue macrophages [1]. The sections were incubated with anti-human (MAC-387 and CD68, 1:75 each) and anti-rat macrophage (ED-1, 1:75) antibodies for $2 \mathrm{~h}$ followed by anti-mouse peroxidase-conjugated secondary antibodies $(1: 100$, DAKO A/S Denmark) for $60 \mathrm{~min}$. The controls included staining the lung sections with only secondary antibody or with rabbit anti-human vonWillebrand Factor antibody (vWF; DAKO A/S, Denmark) which reacts with vWF stored in Weibel-Palade bodies in endothelial cells. This antibody is routinely used to delineate vasculature and as a control in immunostaining protocols [30]. The color was developed with a commercial peroxidase histochemical kit (Vector Laboratories, Canada). Positive cells were counted in 20 oil immersion $(\times 100)$ randomly chosen fields in one section from each of the blocks from each horse.

\subsubsection{Routine electron microscopy}

One-micrometer toluidene blue-stained sections were prepared to select the area of interest for ultra-thin microtomy. The sections $(80-100 \mathrm{~nm})$ were stained with uranyl acetate and lead citrate, and examined in an electron microscope at $80 \mathrm{kV}$.

\subsubsection{Immuno-electron microscopy}

The sections $(100 \mathrm{~nm})$ were blocked with $1 \%$ normal goat serum followed by incubation with MAC-387 antibody (1:50) and $10 \mathrm{~nm}$-gold conjugated goat antimouse secondary antibody (1:100, British Biocell International, United Kingdom). Control sections were stained without the primary antibody and with rabbit anti-vWF antibody. The sections were stained with uranyl acetate and lead citrate and examined in an electron microscope at $80 \mathrm{kV}$.

\subsubsection{Staining for apoptosis}

Lung sections from all the horses were stained using a terminal deoxytransferasemediated dUTP nick-end labeling (TUNEL) kit (Roche Inc., USA) to detect apoptotic cells. This method is based on the specific binding of terminal deoxynucleotidyl transferase (TdT) to the 3-OH ends of DNA. Sections were nick-end labeled with biotinylated dUTP and TdT, and stained with avidin-conjugated peroxidase. The control sections were processed without the inclusion of the TdT buffer.

\subsection{Statistical analyses}

Differences in cell counts between the groups were examined by ranking the variables and then performing a one-way analysis of variance (ANOVA) on the ranks. When significant differences were detected, the mean ranks were compared using the Tukey test. Each of the pressure readings (diastolic, mean and systolic) were summed over the three measurement times and the differences in the sums of the pressures were examined using the Wilcoxin Rank Sum test. Probabilities of less than 5\% ( $p<$ $0.05)$ were considered significant. The statistical analyses were carried out using a commercial software program (Statistix for Windows, version 7, Analytical Software, Box 12185, Tallahassee, FL 323317-2185, USA).

\section{RESULTS}

\subsection{Hematology}

There were no differences in differential blood cell counts between the groups (data not shown). 


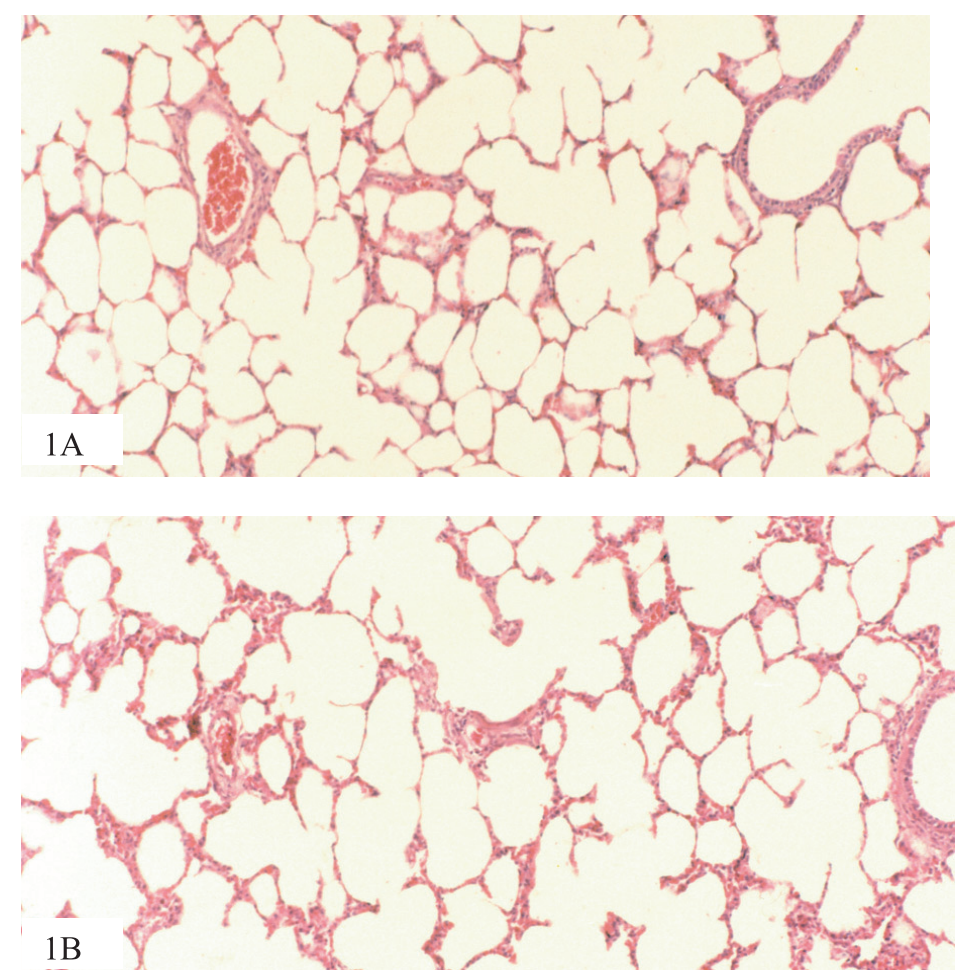

Figure 1. The light micrographs taken from the lung sections from a control (1A) and GC-treated (1B) horse showing normal parenchyma (H \& E staining, $\times 80)$.

\subsection{Physiological response to GC injection}

There were no differences in systolic, diastolic and mean pulmonary arterial pressures between the control and GC-treated horses (data not shown).

\subsection{Histopathology}

Examination of the sections stained with hematoxylin and eosin revealed no signs of lung inflammation in the control as well as GC-treated horses (Fig. 1).

\subsection{Immunohistology}

Serial sections were prepared from the same block for immuno-staining. Lung sections from the control or GC-treated horses did not yield staining when incubated with only secondary antibody (Fig. 2A). However, anti-vWF antibody used as a control delineated blood vessels and some blood cells (Fig. 2B). Although we used three anti-macrophage antibodies (ED-1, MAC387 and CD68, Figs. 2C-2E), only MAC387 antibody stained large septal cells resembling macrophages in an intense and specific manner.

\subsection{Immuno-electron microscopy}

Lung sections stained with the MAC387 antibody and gold-conjugated secondary antibodies showed specific labeling in PIMs but not in microvascular endothelial cells or other vascular cells (Fig. 3A). However, the vWF antibody used as a control 


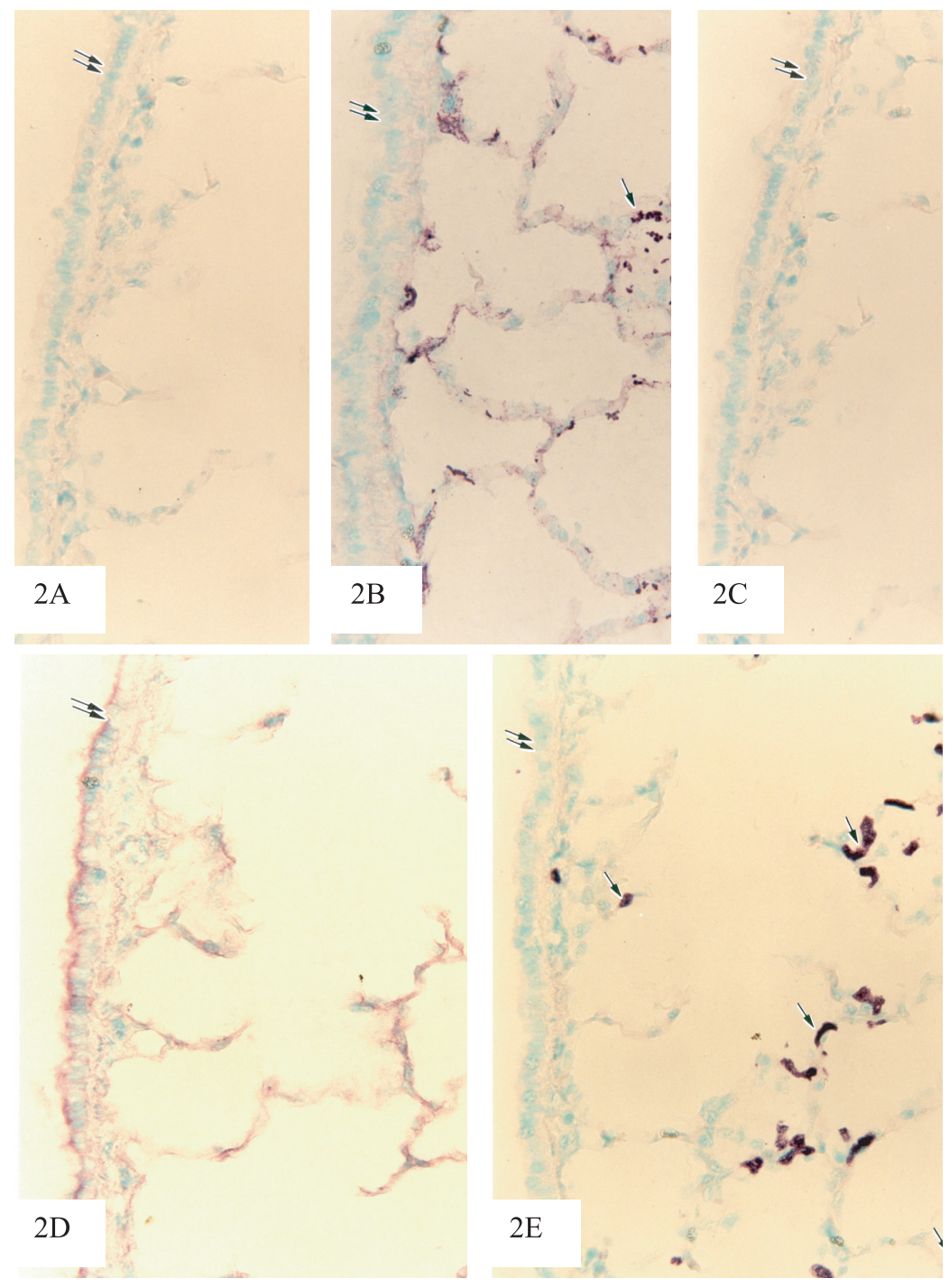

Figure 2. This figure shows light micrographs taken from consecutive lung sections from a control horse. Note the lack of staining following exposure to only secondary antibody (2A) or antibodies against ED-1 (2C) or CD68 (2D). The positive reaction (single arrows) is observed with vWF (2B) and MAC-387 (2E) antibodies. Double arrows: bronchiolar epithelium. $\times 200$.

stained Weibel-Palade bodies in endothelial cells (Fig. 3B).

\subsection{Effect of GC on equine PIMs}

The ability of MAC-387 antibody to recognize PIMs provided us with a tool to pre- cisely quantify the effects of GC on these cells. Horses given a single intravenous injection of GC had lower MAC-387 cell counts at 48 and $72 \mathrm{~h}$ post-treatment (Figs. 4A and 4B). Horses sampled $72 \mathrm{~h}$ after treatment had a median cell count of 2 


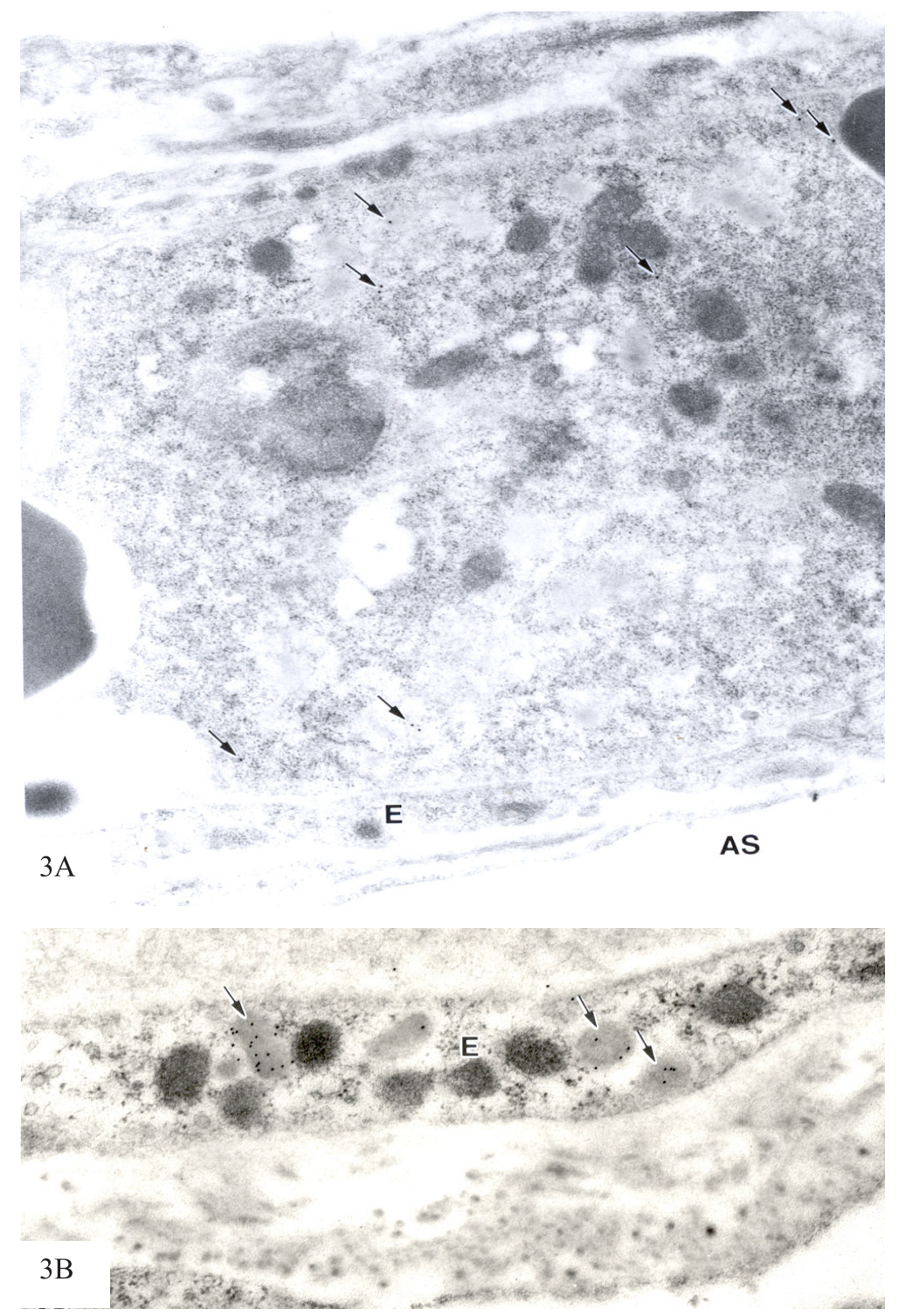

Figure 3. An electron micrograph (3A) from a lung section stained with MAC-387 antibody shows labeling (single arrows) in a PIM while vWF antibody (3B) reacts with Weibel-Palade bodies (single arrows) in an endothelial cell (E). AS: alveolar space. $\times 37500$.

that is significantly less than the median of 9.4 cells observed in the control animals $(p=0.02)($ Fig. 4C).

Since GC activates apoptosis in macrophages in vitro, TUNEL was used to inves- tigate if it induces a similar phenomenon in vivo. There was a trend suggesting an increase in TUNEL-reactive cells at $48 \mathrm{~h}$ and $72 \mathrm{~h}$ post-GC treatment compared to the controls (Figs. 5A and 5B). When 

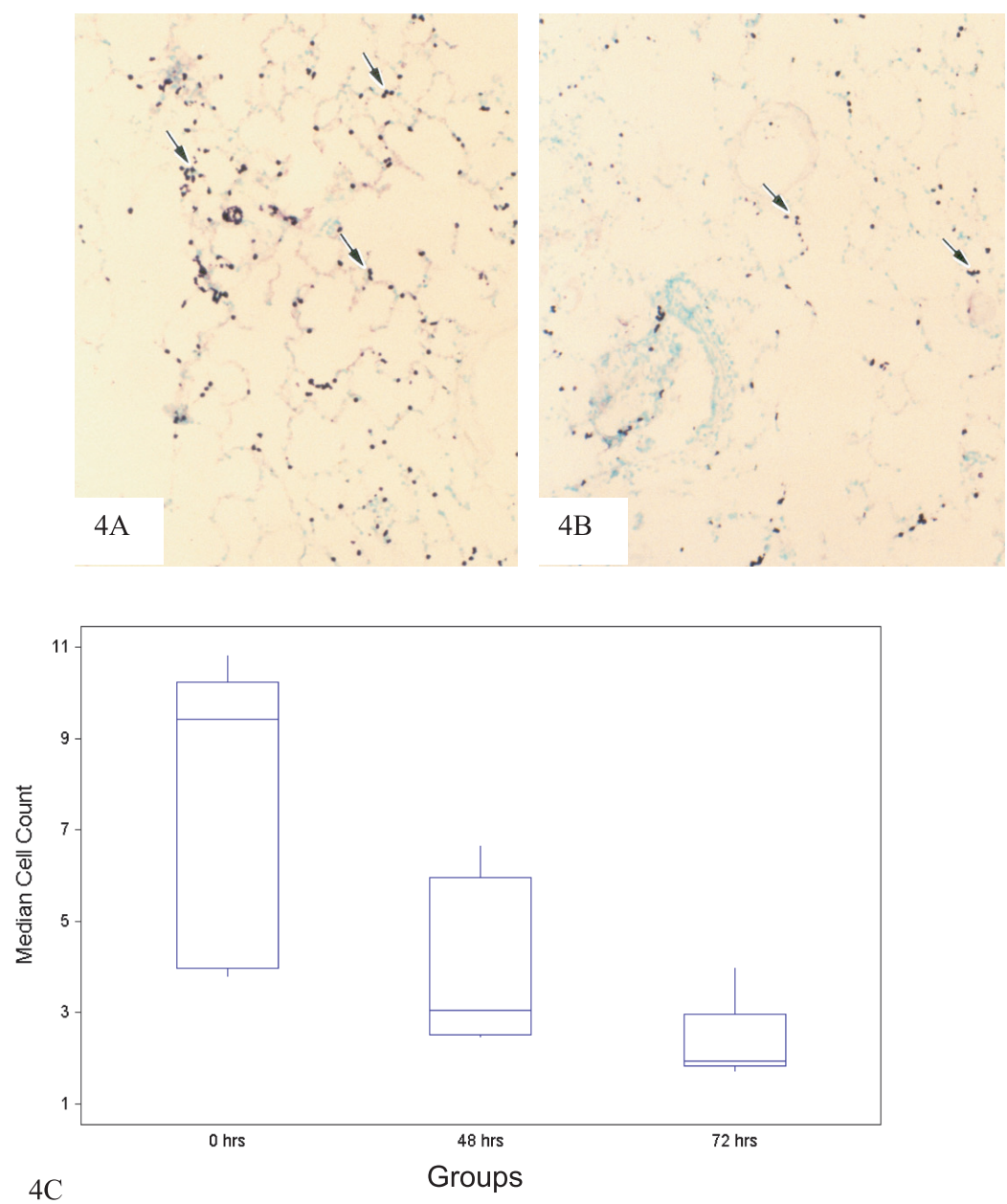

Figure 4. MAC-387-positive cells (single arrows) in lung sections from a control horse (4A) and GC-treated horse (4B). Median MAC-387 positive cell count (4C) in 5 control horses ( 0 h posttreatment), 6 horses ( $48 \mathrm{~h}$ post-treatment) and 4 horses ( $72 \mathrm{~h}$ post-treatment). Boxes enclose the 25 th to 75 th percentile for each group and are bisected at the value for the median. The whiskers indicate the range of "typical" data values. Figures A-B: $\times 40$.

compared with the controls, the median number of reactive cells in the group sampled $72 \mathrm{~h}$ after treatment appeared to be increased but this difference just failed to reach statistic significance $(p=0.07)$ (Fig. 5C). EM confirmed the presence of many apoptotic PIMs in GC-treated horses (Fig. 6). Unlike normal PIMs, the apoptotic PIMs contained apoptotic bodies and con- densed nuclear material as typical ultrastructural features of apoptosis (Fig. 6B).

\section{DISCUSSION}

Macrophages differentiate from monocytes upon migration out of capillaries and, therefore, generally reside in the 

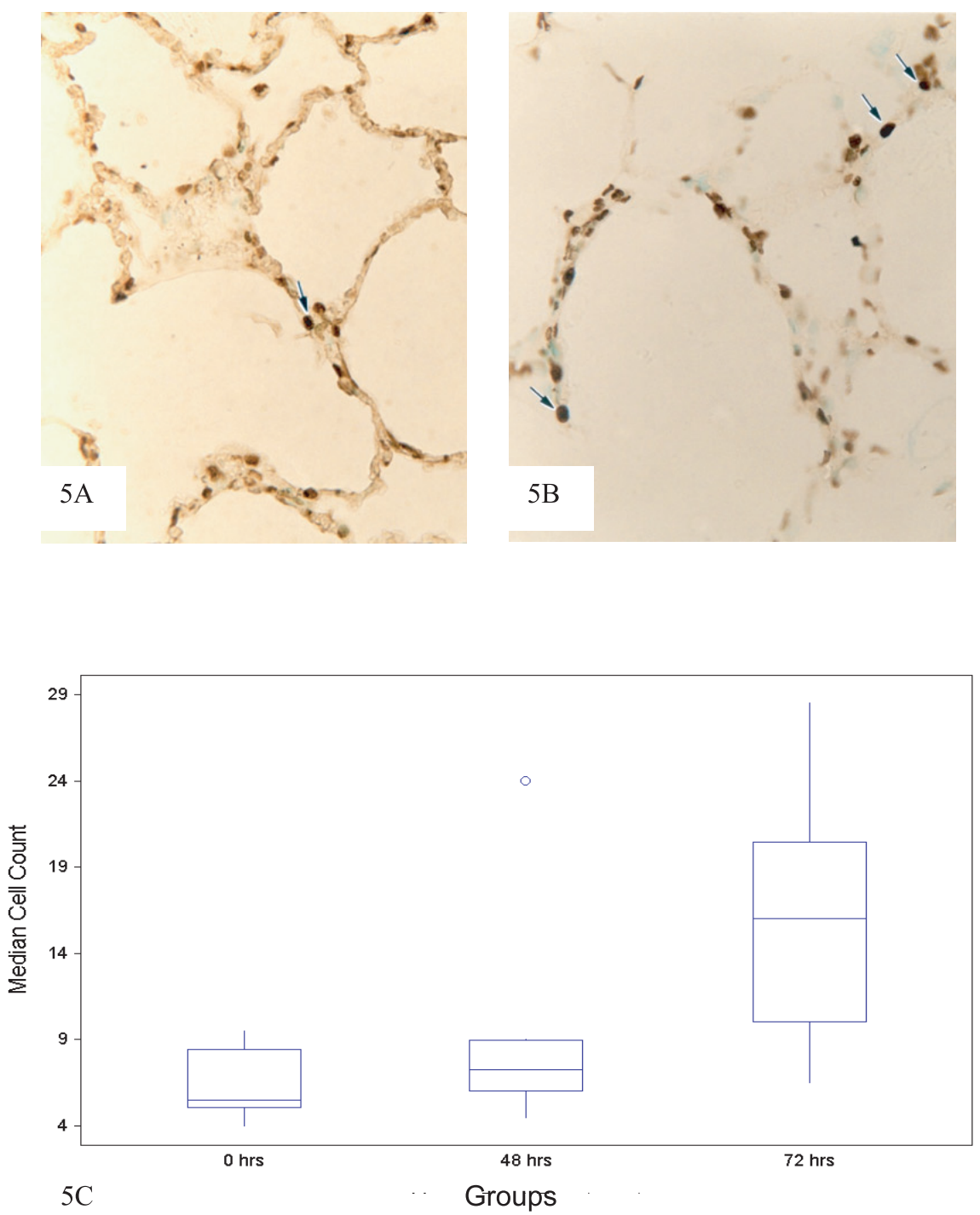

Figure 5. A light micrograph from a lung section from a control horse (5A) shows an occasional TUNEL-positive septal cell (single arrow). Many TUNEL-positive cells (single arrows) in alveolar septae are present in a light micrograph (5B) taken from a section from a GC-treated horse. 5C shows median TUNEL cell counts in 5 control horses $(0 \mathrm{~h}$ post-treatment), 6 horses $(48 \mathrm{~h}$ posttreatment) and 4 horses ( $72 \mathrm{~h}$ post-treatment). Boxes enclose the 25 th to 75 th percentile for each group and are bisected at the value for the median. The whiskers indicate the range of "typical" data values and 0 indicates a probable outlier. Figures A-B: $\times 200$. 

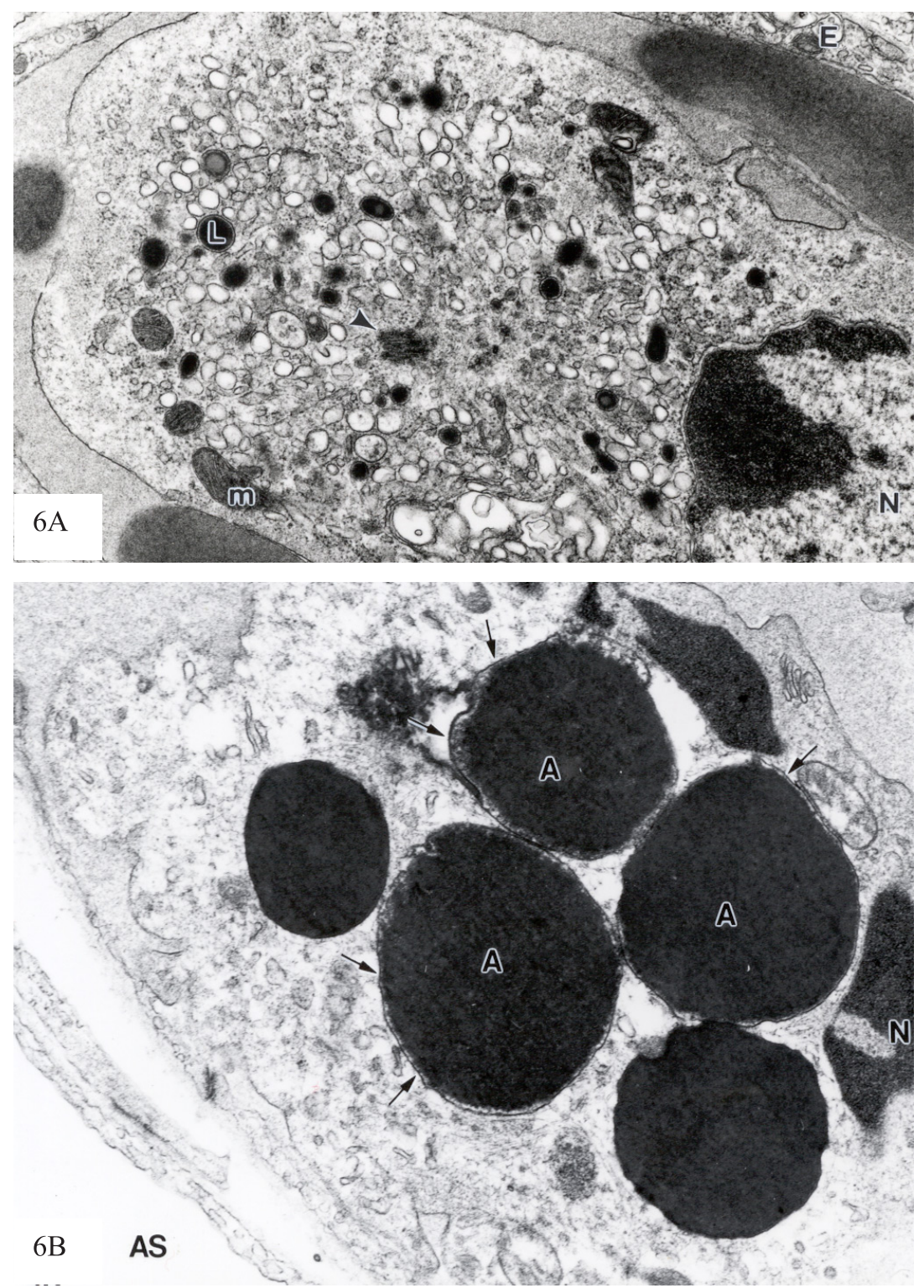

Figure 6. An electron micrograph from a lung section of a control horse (6A) shows a normal PIM with nucleus $(\mathrm{N})$, lysosomes $(\mathrm{L})$, mitochondria $(\mathrm{m})$ and a centriole (arrowhead). The micrograph (6B) taken from a lung section from a GC-treated horse shows an apoptotic PIM with many membrane-bound (single arrows) apoptotic bodies (A) and a remnant of a nucleus $(\mathrm{N})$. AS: alveolar space; E: endothelial cell. $\times 20000$. 
extravascular compartment [13]. There are examples, however, of intravascular macrophages such as those in the liver and the lung [32]. Although liver intravascular macrophages are present in all the known mammalian species, the PIMs are restricted to only a few of the species such as cattle, horses, pigs, sheep and goats [5, 32]. Because the presence or absence of PIMs influences the response of the lung to disease, it is important to study them. Many factors, including a lack of an immuno-phenotypic marker and an inability to isolate equine PIMs for in vitro studies, have hampered the investigation of these cells. Here we report that MAC-387 is a reliable marker for the in situ identification of PIMs and that GC significantly reduces the numbers of PIMs.

Three monoclonal antibodies that identify mononuclear phagocytes in rats and humans have been tested for their reactivity with equine PIMs. Of these, ED-1 and CD68 recognize a $90-110 \mathrm{kDa}$ protein in the lysosomes of rat and human macrophages, respectively, and have been used for immunohistochemistry in the respective species [1, 24]. MAC-387 identifies another cytoplasmic, most probably lysosomal, protein called L1 in the lung and liver macrophages in humans $[11,12]$. These antibodies also show some reactivity against monocytes but very weak reaction with peripheral blood granulocytes. Bovine macrophages including PIMs are stained with CD68 antibody [1, 22]. However, equine PIMs and alveolar macrophages did not stain with either CD68 or ED-1. It is possible that further technical refinements may reveal the usefulness of ED-1 and CD68 to identify lung macrophages including PIMs in horses. However, in our hands, only MAC-387 reacted with equine PIMs, which compared to ED-1, is believed to recognize mature tissue macrophages such as PIMs. Immuno-EM confirmed the localization of MAC-387 in the cytoplasm of equine PIMs. Because we have not tested MAC-387 in other organs such as the liver, our data can only be used for the in situ identification of PIMs.

Many other investigators have also used GC to remove alveolar and liver macrophages $[6,23,28]$. It was reported previously that sheep and calves treated with GC show losses of PIMs [22, 28]. Now GC infusion in horses caused a decline in PIM numbers at 48 and $72 \mathrm{~h}$. Even though the differences between $48 \mathrm{~h}$ and $72 \mathrm{~h}$ post-GC treatment were not significant, the data suggest a steady decline over this period of time. This response was slightly different from that observed in cattle and sheep where an increase was noticed in PIM numbers between $48 \mathrm{~h}$ and $72 \mathrm{~h}$ after GC treatment $[22,28]$. These inter-species differences in the kinetics of PIM depletion not withstanding, GC treatment offers us a large possibility for the investigation of lung pathophysiology in the absence of PIMs and the comparison with normal horses.

Gadolinium chloride affects macrophages because its phagocytosis is a prerequisite for its cellular actions [19]. For example, GC administered intratracheally or intravenously reduces the number of alveolar macrophages and Kupffer cells, respectively, but has no effect on endothelial or epithelial cells in rats [6, 21, 25, 34]. Single intravenous treatment with the GC solution primarily affects PIMs in sheep and cattle while sparing Kupffer cells and lung microvascular endothelial cells [28]. This targeted depletion of PIMs may be due to the preferential uptake of GC during its "first pass" through the lung microvasculature by these cells. Such a possibility is supported by data that more than $90 \%$ of tracers and endotoxins following jugular infusion are phagocytosed by the PIMs [9]. Although we have not examined the effect of GC on liver macrophages in horses, we speculate that these may be unaffected as in sheep [28]. Therefore, GC treatment depletes PIMs and can then be used to investigate lung responses in their absence.

There was a trend indicating an increase, though not significant, in TUNEL-positive 
cells in lung alveolar septae of GC-treated horses. Ultrastructural examination strengthened the TUNEL observations and confirmed that the apoptotic cells were PIMs. The PIMs in GC-treated horses showed classical ultrastructural features of apoptosis including apoptotic bodies and condensation of nuclei while other lung cells were unaffected [14]. These in vivo data are similar to in vitro data on GC-mediated apoptosis in alveolar macrophages reported by Mizgerd et al. [19]. Apoptosis is generally believed to be a non-inflammatory mode of cell death since membrane-bound apoptotic bodies prevent the release of hydrolytic enzymes into extra-cellular space and inflammatory damage to neighboring cells [10]. There was neither a pulmonary hypertensive response during GC infusion nor apparent lung damage in GC-treated horses. Therefore, GC appears to be a safe chemical for the removal of PIMs. Previously published data tantalizingly implicate PIMs as a major component in acute lung inflammation in the host species $[9,17,18,33,35]$. These methods will facilitate investigations of acute lung inflammation in horses with depleted PIMs to establish their contributions in clinical conditions such as heaves and endotoxemia.

\section{ACKNOWLEDGEMENTS}

This research was funded through a grant from the Alberta Agricultural Research Institute, Canada to Dr. Baljit Singh. Dr. Parbhakar is a recipient of an Interprovincial Graduate Student Scholarship from the Western College of Veterinary Medicine. We thank Ms. Sarah Caldwell for assistance with immuno-electron microscopy and Dr. K. Janardhan for help in animal handling.

\section{REFERENCES}

[1] Ackermann M.R., DeBey B.M., Stabel T.J., Gold J.H., Register K.B., Meehan J.T., Distribution of anti-CD68 (EBM11) immunore- activity in formalin-fixed, paraffin-embedded bovine tissues, Vet. Pathol. 31 (1994) 340-348.

[2] Atwal O.S., McDonell W., Staempfli H., Singh B., Minhas K.J., Evidence that halothane anaesthesia induces intracellular translocation of surface coat and Golgi response in equine pulmonary intravascular macrophages, J. Submicrosc. Cytol. Pathol. 26 (1994) 369386.

[3] Atwal O.S., Minhas K.J., Ferenczy B.G., Jassal D.S., Milton D., Mahadevappa V.G., Morphology of pulmonary intravascular macrophages (PIMs) in ruminants: ultrastructural and cytochemical behavior of dense surface coat, Am. J. Anat. 186 (1989) 285-299.

[4] Atwal O.S. Saldanha K.A., Erythrophagocytosis in alveolar capillaries of goat lung: Ultrastructural properties of blood monocytes, Acta Anat. 124 (1985) 245-254.

[5] Atwal O.S., Singh B., Staempfli H., Minhas K.J., Presence of pulmonary intravascular macrophages in the equine lung: Some structuro-functional properties, Anat. Rec. 234 (1992) 540.

[6] Bannenberg G., Lundborg M., Johansson A., Pulmonary macrophage function in systemic gadolinium chloride-pretreated rats, Toxicol. Lett. 80 (1995) 105-107.

[7] Chitko-McKown C.G., Blecha F., Pulmonary intravascular macrophages: a review of immune properties and functions, Ann. Rech. Vet. 23 (1992) 201-214.

[8] Chitko-McKown C.G., Chapes S.K., Brown R.E., Phillips R.M., McKown R.D., Blecha F., Porcine alveolar and pulmonary intravascular macrophages: Comparison of immune functions, J. Leukoc. Biol. 50 (1991) 364372.

[9] Decamp M.M., Warner A.E., Molina R.M., Brain J.D., Hepatic versus pulmonary uptake of particles injected into the portal circulation in sheep, Am. Rev. Resp. Dis. 146 (1992) 224-231.

[10] Fine A., Janssen-Heininger Y., Soultanakis R.P., Swisher S.G., Uhal B.D., Apoptosis in lung pathophysiology, Am. J. Physiol. 279 (2000) L423-L427.

[11] Flavell D.J., Jones D.B., Wright D.H., Identification of tissue histiocytes on paraffin sections by a new monoclonal antibody, J. Histochem. Cytochem. 35 (1987) 1217-1226.

[12] Gutierrez M., Forster F.I., McConnell S.A., Cassidy J.P., Pollock J.M., Bryson D.G., The detection of $\mathrm{CD} 2+, \mathrm{CD} 4+, \mathrm{CD} 8+$ and $\mathrm{WC1} 1$, $\mathrm{T}$ lymphocytes, $\mathrm{B}$ cells and macrophages in fixed and paraffin embedded bovine tissue using range of antigen recovery and signal amplification techniques, Vet. Immunol. Immunopathol. 71 (1999) 321-334. 
[13] Johnson R.B. Jr., Monocytes and macrophages, N. Engl. J. Med. 318 (1988) 747752.

[14] Kerr J.F.R., Wyllie A.H., Currie A.R., Apoptosis: a basic biologic phenomenon with wideranging implications in tissue kinetics, $\mathrm{Br}$. J. Cancer 26 (1972) 239-257.

[15] Longworth K.E., Albertine K.H., Staub N.C., Ultrastructural quantification of pulmonary intravascular macrophages in newborn and 2-week-old lambs, Anat. Rec. 246 (1996) 238-244.

[16] Longworth K.E., Jarvis K.A., Tyler W.S., Steffey E.P. Staub N.C., Pulmonary intravascular macrophages in horses and ponies, Am. J. Vet. Res. 55 (1994) 382-388.

[17] Longworth K.E., Smith B.L., Staub N.C., Steffey E.P., Serikov V.B., Use of detergent to prevent initial responses to endotoxin in horses, Am. J. Vet. Res. 57 (1996) 10631066.

[18] Miyamoto K., Schultz E., Heath T., Mitchell M.D., Albertine K.H., Staub N.C., Pulmonary intravascular macrophages and hemodynamic effects of liposomes in sheep, J. Appl. Physiol. 64 (1988) 1143-1152.

[19] Mizgerd J.P., Molina R.M., Stearns R.C., Brain J.D., Warner A.E., Gadolinium induces macrophage apoptosis, J. Leukoc. Biol. 59 (1996) 189-195.

[20] Morris D.D., Endotoxemia in horses: A review of cellular and humoral mediators involved in its pathogenesis, J. Vet. Intern. Med. 5 (1991) 167-181.

[21] Olynyk J.K., Yeoh G.C., Ramm G.A., Clarke S.L., de la Hall P., Britton R.S., Bacon B.R., Tracy T.F., Gadolinium chloride suppresses hepatic oval cell proliferation in rats with biliary obstruction, Am. J. Pathol. 152 (1998) 347-352.

[22] Pearce J.W., Gamage L., Singh B., Pulmonary intravascular macrophages promote acute lung inflammation, FASEB J. 15 (2001) A57.

[23] Pendino K.J., Meidhof T.M., Heck D.E., Laskin J.D., Laskin D.L., Inhibition of macrophages with gadolinium chloride abrogates ozone-induced pulmonary injury and inflammatory mediator production, Am. J. Respir. Cell Mol. Biol. 13 (1995) 125-132.

[24] Pulford K.A.F., Sipos A., Cordell J.L., Stross W.P., Mason D.Y., Distribution of the CD68 macrophage/myeloid associated antigen, Int. Immunol. 2 (1990) 973-980.

[25] Rai R.M., Loffreda S., Karp C.L., Yang S.-Q., Lin H.-Z., Diehl A.M., Kupffer cell depletion abolishes induction of interleukin-10 and permits sustained overexpression of tumor necrosis factor alpha messenger RNA in the regenerating rat liver, Hepatology 25 (1997) 889-895.
[26] Rogers R.A., Tasat D.R., Warner A.E., Brain J.D., Quantitative recovery of pulmonary intravascular macrophages from sheep lungs, J. Leukoc. Biol. 56 (1994) 692-701.

[27] Singh B., Atwal O.S., Ultrastructural and immunocytochemical study of the pulmonary intravascular macrophages of Escherichia coli lipopolysaccharide-treated sheep, Anat. Rec. 247 (1997) 214-224.

[28] Singh B., de la Concha-Bermejillo A., Gadolinium chloride removes pulmonary intravascular macrophages and curtails the degree of ovine lentivirus-induced lymphoid interstitial pneumonia, Int. J. Exp. Pathol. 79 (1998) 151-162.

[29] Singh B., Minhas K.J., Atwal O.S., Ultracytochemical study of multiple dose effect of monastral blue uptake by equine pulmonary intravascular macrophages (PIMs), J. Submicroc. Cytol. Pathol. 26 (1994) 235-243.

[30] Singh B., Rawlings N., Kaur A., Expression of integrin $\alpha v \beta 3$ in pig, dog and cattle, Histol. Histopathol. 16 (2001) 1037-1046.

[31] Sone Y., Serikov V.B., Staub N.C., Intravascular macrophage depletion attenuates endotoxin lung injury in anesthetized sheep, J. Appl. Physiol. 87 (1999) 1354-1359.

[32] Staub N.C., Pulmonary intravascular macrophages, Annu. Rev. Physiol. 56 (1994) 4767.

[33] Staub N.C., Longworth K.E., Serikov V.B., Jerome E.H., Elsasser T.H., Detergent inhibits $70-90 \%$ of responses to intravenous endotoxin in awake sheep, J. Appl. Physiol. 90 (2001) 1788-1797.

[34] Thepen T., van Rooijen N., Kraal G., Alveolar macrophage elimination in vivo is associated with an increase in pulmonary immune response in mice, J. Exp. Med. 170 (1989) 499-509.

[35] Warner A.E., Pulmonary intravascular macrophages: Role in acute lung injury, Clin. Chest Med. 17 (1996) 125-135.

[36] Warner A.E., Decamp M.M., Molina R.M., Brain J.D., Pulmonary removal of circulating endotoxin results in acute lung injury in sheep, Lab. Investig. 59 (1988) 219-230.

[37] Winkler G.C., Pulmonary intravascular macrophages in domestic animal species: Review of structural and functional properties, Am. J. Anat. 181 (1988) 217-234.

[38] Winkler G.C., Cheville N.F., Monocytic origin and postnatal mitosis of intravascular macrophages in the porcine lung, J. Leukoc. Biol. 38 (1985) 471-480.

[39] Yoneda S., Emi N., Fujita Y., Ohmichi M., Hirano S., Suzuki K.T., Effects of gadolinium chloride on the rat lung following intratracheal instillation, Fundam. Appl. Toxicol. 28 (1995) 65-70. 